\title{
Evaluation of HRCT for the Differential Diagnosis of Pneumonia-Like Consolidation Pattern of Pulmonary MALT Lymphoma from Lobar Pneumonia
}

\author{
Wenli Qiu' \\ Haibin Chen ${ }^{2}$ \\ Jian Zhang ${ }^{3}$ \\ Ren Shuai ${ }^{1}$ \\ Huifeng Zhang' \\ Kai Guo' \\ Li Zhu' \\ Zhongqiu Wang' \\ 'Department of Radiology, Affiliated \\ Hospital of Nanjing University of Chinese \\ Medicine, Nanjing, Jiangsu, People's \\ Republic of China; ${ }^{2}$ Science and \\ Technology Department, Jiangsu \\ Collaborative Innovation Center of \\ Traditional Chinese Medicine Prevention \\ and Treatment of Tumor, Nanjing \\ University of Chinese Medicine, Nanjing, \\ jiangsu, People's Republic of China; \\ ${ }^{3}$ Department of Radiology, Liyang \\ Traditional Chinese Medicine Hospital, \\ Changzhou, Jiangsu, People's Republic of \\ China
}

Purpose: To assess the performance of high-resolution computed tomography (HRCT) in discriminating the consolidation pattern of pulmonary mucosa-associated lymphoid tissue (MALT) lymphoma from lobar pneumonia.

Patients and Methods: This retrospective study comprised 26 patients with pathologically confirmed consolidation pattern of pulmonary MALT lymphoma (12 men and 14 women; mean age, $59.4 \pm 12.1$ years) and 36 patients with lobar pneumonia confirmed by body fluids or respiratory secretion culture (16 men and 20 women; mean age, $41.8 \pm 26.3$ years). Two radiologists independently evaluated the CT images. The effectiveness of these variables in distinguishing lobar pneumonia from MALT lymphoma was analyzed using logistic regression analysis.

Results: The average age of lobar pneumonia patients was younger than that of MALT lymphoma patients $(p=0.002)$. The respiratory symptom was more common in lobar pneumonia than MALT lymphoma $(p=0.002)$. Signs of bronchiectasis within the consolidation and bulging of interlobar fissure occurred significantly more often in MALT lymphoma than pneumonia $(69.2 \%$ vs $11.1 \%, p<0.0001 ; 46.2 \%$ vs $19.4 \%, p=0.024)$. We used the predictors with $p<0.05$ (age, respiratory symptoms, bronchiectasis, and bulging of interlobar fissure) to construct a logistic regression model. The area under curve (AUC), negative predictive value (NPV), positive predictive value (PPV), sensitivity, specificity, and accuracy were 0.891 , $84.21 \%, 83.33 \%, 88.89 \%, 76.92 \%$, and $83.87 \%$ for discriminating lobar pneumonia from MALT lymphoma.

Conclusion: Middle-aged, presence of mild clinical symptoms, bronchiectasis and bulging of the interlobar fissure on chest CT images are potential markers to distinguish pulmonary MALT lymphoma from lobar pneumonia.

Keywords: high-resolution computed tomography, pulmonary mucosa-associated lymphoid tissue lymphoma, lobar pneumonia, diagnosis

\section{Introduction}

Primary pulmonary lymphoma (PPL) is a rare type of extranodal lymphoma, and is defined as lymphoid proliferation involving bronchi and/or lung parenchyma with no evidence of extra pulmonary lymphoma within 3 months of initial diagnosis. ${ }^{1}$ PPL accounts for $0.4 \%$ of all lymphomas, $0.5 \%$ of the primary pulmonary malignancies, and $3.6 \%$ of all extranodal lymphomas. Pulmonary mucosa-associated lymphoid tissue (MALT) lymphoma is the most common type of PPL, also known as extra nodal marginal zone B cell lymphoma, which can arise from any mucosal site. Among them, the lungs are the commonly involved organs, especially
Correspondence: Zhongqiu Wang Department of Radiology, Affiliated Hospital of Nanjing University of Chinese Medicine, 155 Hanzhong Road, Nanjing, 210029, Jiangsu Province, People's Republic of China

Tel +8625886I 8472

Fax +862586618139

Email zhq200105@sina.com 
the MALT of the bronchus. ${ }^{2}$ Some patients with pulmonary MALT lymphoma may have chest pain, cough, mild dyspnea, and hemoptysis. However, all these symptoms are not specific, and some patients are asymptomatic. ${ }^{3}$ Pulmonary MALT lymphoma remains a diagnostic challenge in clinic, and histologic confirmation is mandatory for definitive diagnosis of this disease.

Computed tomography (CT) is widely used to evaluate pulmonary lesions, and the imaging manifestations of pulmonary MALT lymphoma observed on CT scans are diversiform. ${ }^{4}$ Based on the pattern of parenchymal abnormalities, pulmonary MALT lymphoma is classified into four types: pneumonia-like consolidation pattern, nodular or mass pattern, ground-glass opacity pattern, and diffuse interstitial lung disease pattern. ${ }^{5}$ Among them, consolidation is the most common pattern with lesions present consolidation in polygon-based pleura consolidation involving lobe/segment. Similar to lobar pneumonia, consolidation pattern can be easily misdiagnosed as pneumonia. ${ }^{6,7}$ Pulmonary MALT lymphoma is usually treated with chemotherapy, immunotherapy or surgery, while lobar pneumonia is usually treated with antibiotics. $^{8}$ Based on the fact, it is important to distinguish MALT lymphoma from lobar pneumonia accurately in order to institute appropriate treatment to improve their prognosis.

Although pulmonary MALT lymphoma has been reported from clinical, pathologic and radiological imaging perspectives, few reports have focused on its differential diagnosis from lobar pneumonia. Thus, the aim of the study was to retrospectively evaluate whether consolidation pattern of pulmonary MALT lymphoma can be distinguished from lobar pneumonia on the basis of CT imaging features and clinical characteristics.

\section{Patients and Methods}

The retrospective study was approved by the Institutional Review Board for Health Sciences Research at the Affiliated Hospital of Nanjing University of Chinese Medicine. Due to the retrospective, observational, and anonymous nature of the study, informed consent for was waived. The work has been carried out in accordance with the Declaration of Helsinki.

\section{Patients}

From September 2014 to October 2020, 69 consecutive patients with pathologically confirmed pulmonary MALT lymphoma were identified from our medical records. The diagnosis of pulmonary MALT lymphoma was based on surgical resection $(\mathrm{n}=27)$, CT-guided percutaneous lung biopsy $(\mathrm{n}=31)$, and bronchoscopic biopsy $(\mathrm{n}=11)$. Inclusion criteria were as follows: Chest CT scan showed patchy consolidation of lung parenchyma. The lesions presented consolidation in polygon-based pleura consolidation involving lobe/segment. The exclusion criteria were as follows: 1) patient with incomplete histopathologic evaluation $(\mathrm{n}=9) ; 2)$ suboptimal image quality $(\mathrm{n}=2)$; 3)the morphological pattern was nodule, mass, and ground-glass opacity $(\mathrm{n}=25)$; 4) chemotherapy therapy before CT examination $(n=7)$. As a result, a total of 26 patients $(12$ men and 14 women; mean age, $59.4 \pm 12.1$ years; age range, $32-78$ years) were enrolled in MALT group (Figure 1).

Similarly, from April 2017 to December 2020, 58 consecutive patients with lobar pneumonia confirmed by body fluids or respiratory secretion culture were identified from our medical records. Inclusion criteria were as follows: 1) chest CT scan showed patchy consolidation of lung parenchyma and 2) the lesions presented consolidation in polygon-based pleura consolidation involving lobe/segment. The exclusion criteria were as follows: 1) patient with negative of body fluids or respiratory secretion culture $(\mathrm{n}=7) ; 2)$ anti-inflammatory therapy is ineffective $(\mathrm{n}=2) ; 3)$ suboptimal imaging quality $(n=4)$; 4) previous history of steroid or immunosuppressant therapy before CT scan $(n=9)$. As a result, a total of 36 patients (16 men and 20 women; mean age, $41.8 \pm 26.3$ years; age range, 4-76 years) were enrolled in lobar pneumonia group (Figure 1).

\section{CT Technique}

All patients underwent chest CT scanning using Light Speed 64VCT (GE Healthcare) and Brilliance 64 (Phillips Healthcare) following the standardized protocol. Scanning was performed from the top of the thoracic cavity to the level of bilateral adrenal glands. Helical scanning protocol was as follows: $120 \mathrm{kVp}$; 70-200 mAs; beam pitch, 0.516-0.98; slice thickness, $5 \mathrm{~mm}$; slice interval, $5 \mathrm{~mm}$; matrix, $512 \times 512$. Sections of $1 \mathrm{~mm}$ thickness were reconstructed with a sharp reconstruction algorithm. All images were displayed in both lung window (window width, $1500 \mathrm{HU}$; window level, -700 HU) and mediastinal window (window width, $400 \mathrm{HU}$; window level, $20 \mathrm{HU})$.

\section{Imaging Analysis}

All the chest CT images were evaluated by two independent experienced thoracic radiologists (WQ and HZ) with more 
Chest CT scan showed patchy consolidation of lung parenchyma. The lesions presented consolidation in polygon-based pleura consolidation involving lobe/segment

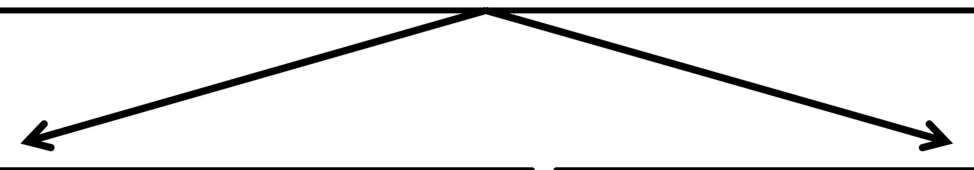

Patients with pathologic diagnoses of pulmonary MALT lymphoma by CT-guided percutaneous lung biopsy, bronchoscopic biopsy, and surgical resection between September 2014 and October 2020. $(n=69)$

Exclude:

- with incomplete histopathologic evaluation $(\mathrm{n}=9)$

- suboptimal imaging quality $(\mathrm{n}=2)$

- The morphological pattern was nodule, mass, and ground glass opacity $(\mathrm{n}=25)$

- chemotherapy therapy before CT examination $(\mathrm{n}=7)$

26 patients with pulmonary

MALT lymphoma were included
Patient with body fluids or respiratory secretions culture proved lobar pneumonia between April 2017 and December 2020. $(\mathrm{n}=58)$

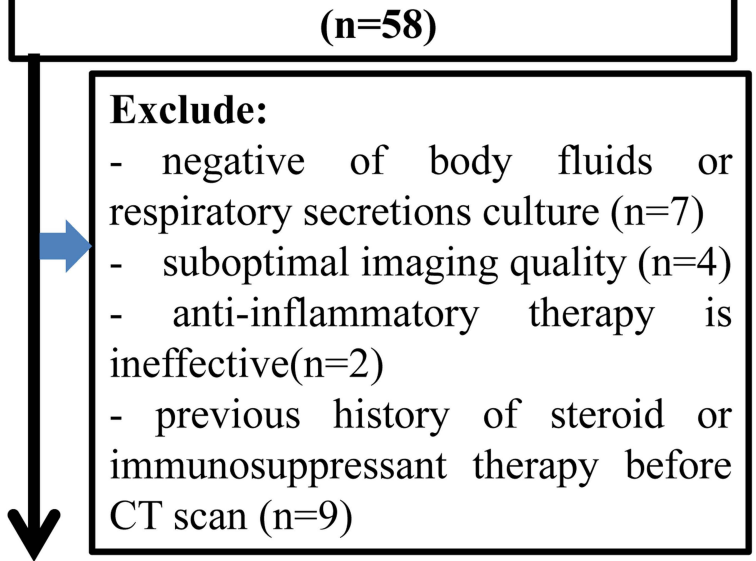

36 patients with lobar pneumonia were included

Figure I Flow diagram of patients' selection.

Abbreviations: CT, computed tomography; MALT, mucosa-associated lymphoid tissue.

than 5 years of experience. If disparities in opinion were encountered, a consensus opinion was obtained by discussion with a senior radiologist $(\mathrm{ZW})$ with more than 30 years of experience. The observers were blinded to the laboratory, clinical, and histopathological outcome. The following CT imaging features were evaluated: lung lesion distribution, location, size, air bronchogram sign, bronchiectasis and calcification within the consolidation, bulging of interlobar fissure, mediastinum or hilar lymph nodes enlargement (shorter diameter $>10 \mathrm{~mm}$ ), and pleural effusion.

\section{Statistical Analysis}

Data were analyzed using a software package SPSS22.0 (IBM, Chicago, IL, USA). The continuous data were compared by Student's $t$-test, and categorical data were compared by Chi-square test. Statistical significance was denoted as $\mathrm{p}<0.05$. Logistic regression was used to analyze the effectiveness of variables with statistical significance for distinguishing lobar pneumonia from MALT lymphoma and construct different predicting models. Receiver operating characteristic (ROC) curve was constructed, and the area under curve (AUC), negative predictive value (NPV), positive predictive value (PPV), sensitivity, specificity, and accuracy were used to validate the performance of these models.

\section{Results \\ Clinical Characteristics}

The clinical characteristics of 26 MALT lymphoma and 36 lobar pneumonia patients are described in Table 1. There was no significant difference in gender between the two groups. The average age of lobar pneumonia patients was younger than that of MALT lymphoma patients $[41.8 \pm 26.3$ (4-76) vs $59.4 \pm 12.1(32-78)]$ with a $p$-value of 0.002 . The respiratory symptoms such as fever, cough, expectoration, dyspnea, chest pain, and chest distress were more common 
Table I Clinical Features of Patients

\begin{tabular}{|l|l|l|l|}
\hline $\begin{array}{l}\text { Clinical } \\
\text { Characteristics }\end{array}$ & $\begin{array}{l}\text { MALT } \\
(\mathbf{n = 2 6 )}\end{array}$ & $\begin{array}{l}\text { Pneumonia } \\
\mathbf{n = 3 6 )}\end{array}$ & p-value \\
\hline $\begin{array}{l}\text { Age (years) } \\
\text { Mean } \pm \text { SD } \\
\text { (Range) }\end{array}$ & $\begin{array}{l}59.4 \pm 12.1 \\
(32-78)\end{array}$ & $\begin{array}{l}41.8 \pm 26.3 \\
(4-76)\end{array}$ & $0.002^{* *}$ \\
\hline $\begin{array}{l}\text { Gender, n (\%) } \\
\text { Male } \\
\text { Female }\end{array}$ & $12(46.2)$ & $16(44.4)$ & 0.894 \\
\hline $\begin{array}{l}\text { Symptoms } r, \mathrm{n}(\%) \\
\text { Respiratory Symptoms }\end{array}$ & $14(53.8)$ & $20(55.6)$ & \\
Asymptomatic & $13(50.0)$ & $31(86.1)$ & $0.002 * *$ \\
\hline
\end{tabular}

Note: $*^{*} \mathrm{p}<0.01$.

Abbreviations: MALT, mucosa-associated lymphoid tissue; SD, standard deviation.

in lobar pneumonia patients than MALT lymphoma patients $(86.1 \%$ vs $50.0 \%, p=0.002)$.

\section{CT Manifestations}

CT manifestations of MALT lymphoma and lobar pneumonia are summarized in Table 2. A total of 42 lesions were observed in 26 pulmonary MALT lymphoma patients, including 21 with unilateral lesions and 5 with bilateral lesions. A total of 38 lesions were observed in 36 lobar pneumonia patients, including 34 with unilateral lesions and 2 with bilateral lesions. The distribution of lesions was random, without statistical difference between lobes ( $p=$ 0.634). There were no significant statistical differences between MALT lymphoma (Figures 2-4) and lobar pneumonia (Figure 5) patients regarding lesion size, air bronchogram sign, calcification (Figure 2), lymph node enlargement, and pleural effusion $(p>0.05)$. Bronchiectasis within the consolidation was more frequent in MALT lymphoma patients than in lobar pneumonia patients [69.2\% (18 of 26) vs $11.1 \%$ (4 of 36), $p<0.001$ ] (Figures 2 and 3). Cystic bronchiectasis was observed in 11 patients with MALT lymphoma group. In these patients, the dilated cystic bronchus might contain air, liquid, or both, with an airliquid level (Figure 3). Bulging of interlobar fissure was more common in MALT lymphoma patients than in lobar pneumonia patients [46.2\% (12 of 26) vs $19.4 \%$ (7 of 36$)$ ] with a p-value of 0.024 (Figure 4).

\section{Diagnostic Performance}

We used predictors with $\mathrm{p}<0.05$ in univariate analysis to construct three logistic regression models to distinguish lobar pneumonia from MALT lymphoma: model
Table 2 Chest CT Findings and Features of Patients

\begin{tabular}{|c|c|c|c|}
\hline CT Features & $\begin{array}{l}\text { MALT } \\
(n=26)\end{array}$ & $\begin{array}{l}\text { Pneumonia } \\
(n=36)\end{array}$ & p-value \\
\hline $\begin{array}{l}\text { Distribution, n (\%) } \\
\text { Laterality } \\
\text { Unilateral } \\
\text { Bilateral } \\
\text { Location } \\
\text { Right upper lobe } \\
\text { Right middle lobe } \\
\text { Right lower lobe } \\
\text { Left upper lobe } \\
\text { Left lower lobe }\end{array}$ & $\begin{array}{l}21(80.8) \\
5(19.2) \\
12(28.6) \\
7(16.7) \\
7(16.7) \\
9(21.4) \\
7(16.7)\end{array}$ & $\begin{array}{l}34(94.4) \\
2(5.6) \\
15(39.5) \\
7(18.4) \\
4(10.5) \\
9(23.7) \\
3(7.9)\end{array}$ & 0.634 \\
\hline $\begin{array}{l}\text { Lesion size }\left(\mathrm{cm}^{2}\right) \\
\text { Mean } \pm \mathrm{SD} \\
\text { (range) }\end{array}$ & $\begin{array}{l}39.5 I \pm 25.68 \\
(9.24-102.60)\end{array}$ & $\begin{array}{l}42.83 \pm 25.16 \\
(7.99-100.48)\end{array}$ & 0.613 \\
\hline $\begin{array}{l}\text { Air bronchogram sign, } \\
\text { n (\%) } \\
\text { Yes } \\
\text { No }\end{array}$ & $\begin{array}{l}23(88.5) \\
3(11.5)\end{array}$ & $\begin{array}{l}34(94.4) \\
2(5.6)\end{array}$ & 0.393 \\
\hline $\begin{array}{l}\text { Bronchiectasis, n (\%) } \\
\text { Yes } \\
\text { No }\end{array}$ & $\begin{array}{l}18(69.2) \\
8(30.8)\end{array}$ & $\begin{array}{l}4(11.1) \\
32(88.9)\end{array}$ & $<0.00 I^{* * *}$ \\
\hline $\begin{array}{l}\text { Calcification } \\
\text { Yes } \\
\text { No }\end{array}$ & $\begin{array}{l}2(7.7) \\
24(92.3)\end{array}$ & $\begin{array}{l}0(0.0) \\
36(100.0)\end{array}$ & 0.091 \\
\hline $\begin{array}{l}\text { Bulging of interlobar } \\
\text { fissure, n (\%) } \\
\text { Yes } \\
\text { No }\end{array}$ & $\begin{array}{l}12(46.2) \\
14(53.8)\end{array}$ & $\begin{array}{l}7(19.4) \\
29(80.6)\end{array}$ & $0.024 *$ \\
\hline $\begin{array}{l}\text { Lymph node } \\
\text { enlargement, n (\%) } \\
\text { Yes } \\
\text { No }\end{array}$ & $\begin{array}{l}2(7.7 \%) \\
24(92.3 \%)\end{array}$ & $\begin{array}{l}8(22.2) \\
28(77.8)\end{array}$ & 0.125 \\
\hline $\begin{array}{l}\text { Pleural effusion, } n \text { (\%) } \\
\text { Yes } \\
\text { No }\end{array}$ & $\begin{array}{l}4(15.4) \\
22(84.6)\end{array}$ & $\begin{array}{l}12(33.3 \%) \\
24(66)\end{array}$ & 0.111 \\
\hline
\end{tabular}

Notes: ${ }^{*} p<0.05 ; * * * p<0.001$.

Abbreviations: MALT, mucosa-associated lymphoid tissue; SD, standard deviation.

A (clinical characteristic-based model), age+respiratory symptoms; model B (CT manifestation-based model), bronchomatic + bulging of interlobar fissure; model $\mathrm{C}$ (model $\mathrm{A}+\mathrm{B})$, age + respiratory symptoms+bronchomatic + bulging of interlobar fissure. ROC curve was used to measure their diagnostic performances in differentiating lobar pneumonia from MALT lymphoma (Figure 6). As shown in Table 3, the AUC value for model A was 0.765, while its NPV, PPV, sensitivity, 


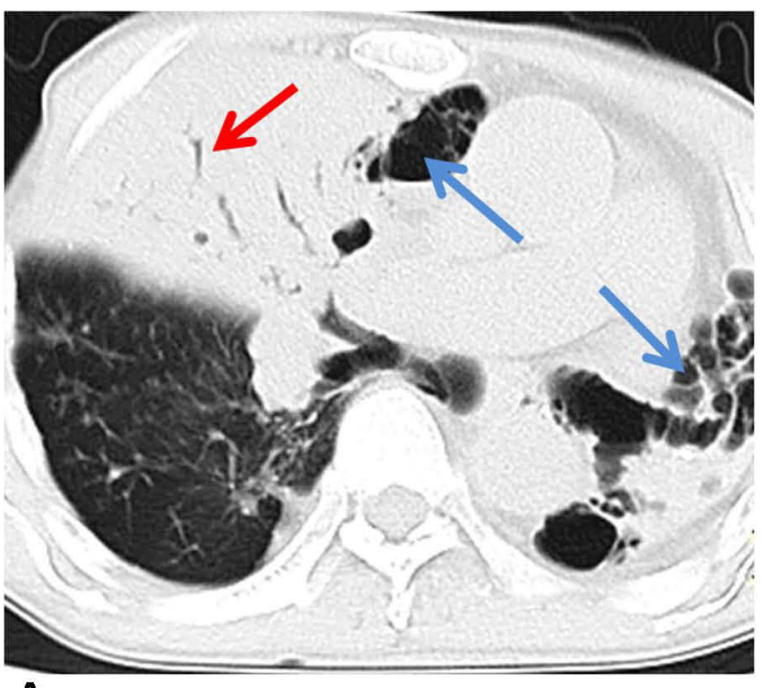

A

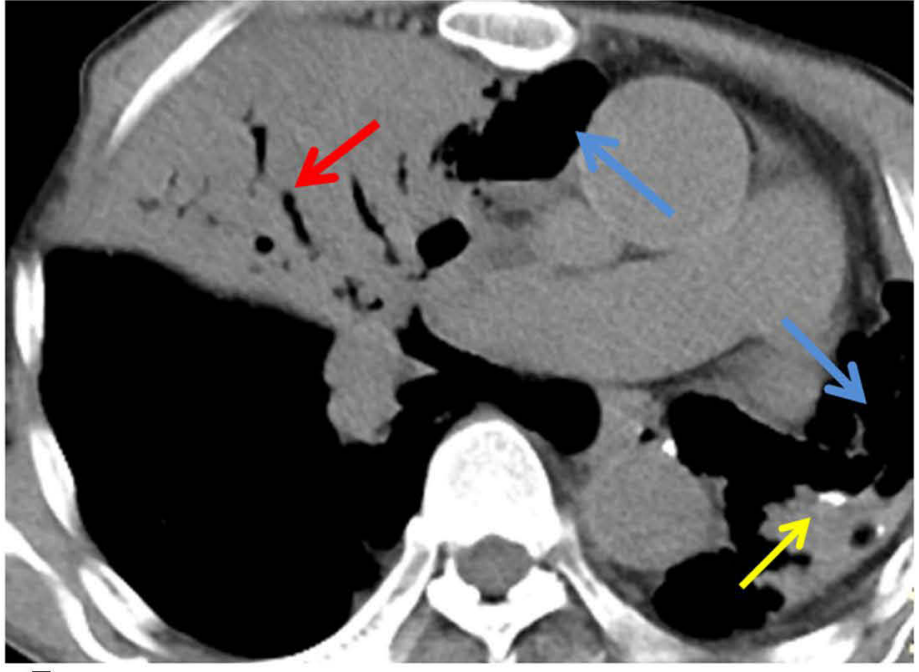

B

Figure 2 Pulmonary MALT lymphoma in a 65-year-old man. (A) Axial lung-window HRCT showed consolidation with air bronchogram (red arrow). There was cystic bronchiectasis (blue arrow) within the lesion in the right middle lobe and left lower lobe. (B) Mediastinum-window showed consolidation with air bronchogram (red arrow) and cystic bronchiectasis (blue arrow), and spot calcification in the lesion (yellow arrow).

Abbreviations: HRCT, high-resolution computed tomography; MALT, mucosa-associated lymphoid tissue.

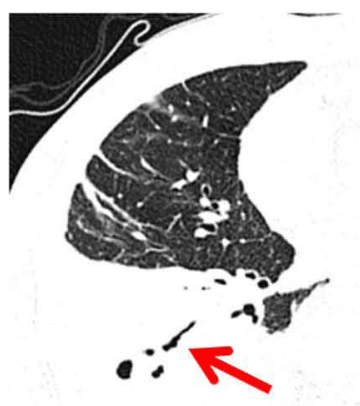

$\triangle$

A
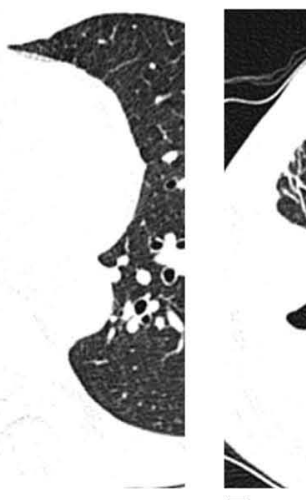

B
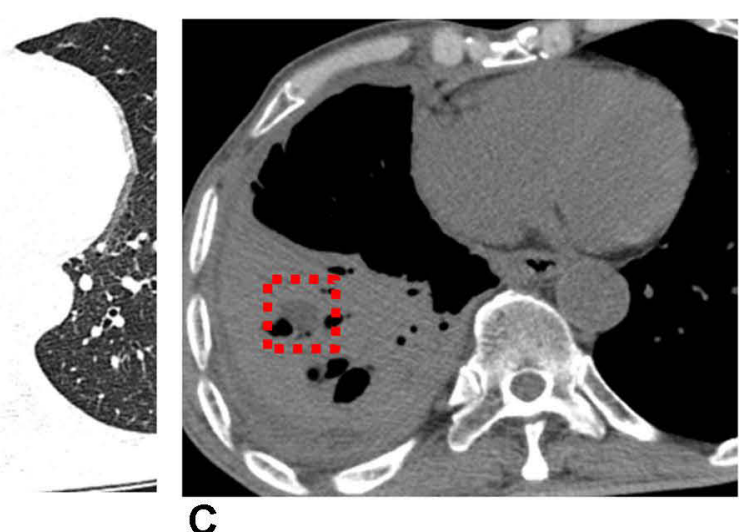

Figure 3 Pulmonary MALT lymphoma in a 7I-year-old man. (A) CT imaging showed consolidation with air bronchogram (red arrow) in the right lower lobe. (B) There was cystic bronchiectasis (blue arrow) in the area of consolidation. (C) Mediastinum-window showed the cyst of the dilated bronchus contained fluid (red dashed box) and little pleural effusion on the right.

Abbreviations: CT, computed tomography; MALT, mucosa-associated lymphoid tissue.

specificity, and accuracy were $71.43 \%, 70.00 \%, 83.33 \%$, $53.85 \%$, and $70.97 \%$, respectively. The AUC value for model B was 0.826 , while its NPV, PPV, sensitivity, specificity, and accuracy were $80.00 \%, 81.82 \%, 88.89 \%$, $69.23 \%$, and $80.65 \%$, respectively. The AUC of model C was 0.891 , with $84.21 \%$ NPV, $83.33 \%$ PPV, $88.89 \%$ sensitivity, $76.92 \%$ specificity, and $83.87 \%$ accuracy for discriminating lobar pneumonia from MALT lymphoma.

\section{Discussion and Conclusion}

Although pulmonary MALT lymphoma is a rare disease, it is the most common type of PPL, accounting for $90 \%$ of
PPL. ${ }^{9}$ Pulmonary MALT lymphoma is often delayed in diagnosis or misdiagnosed due to lack of specific clinical manifestations, biological markers, and characteristic imaging features. ${ }^{10}$ The most frequent pattern of pulmonary MALT lymphoma is consolidation pattern, which is similar to lobar pneumonia in CT imaging. ${ }^{11}$ Misdiagnosis of lobar pneumonia as MALT lymphoma may result in overtreatment, and misdiagnosis of MALT lymphoma as lobar pneumonia may result in delayed chemotherapy or surgical treatment. ${ }^{12}$ In the present study, we analyzed the CT imaging features and clinical characteristics of 26 patients with pulmonary MALT lymphoma and 36 patients with 


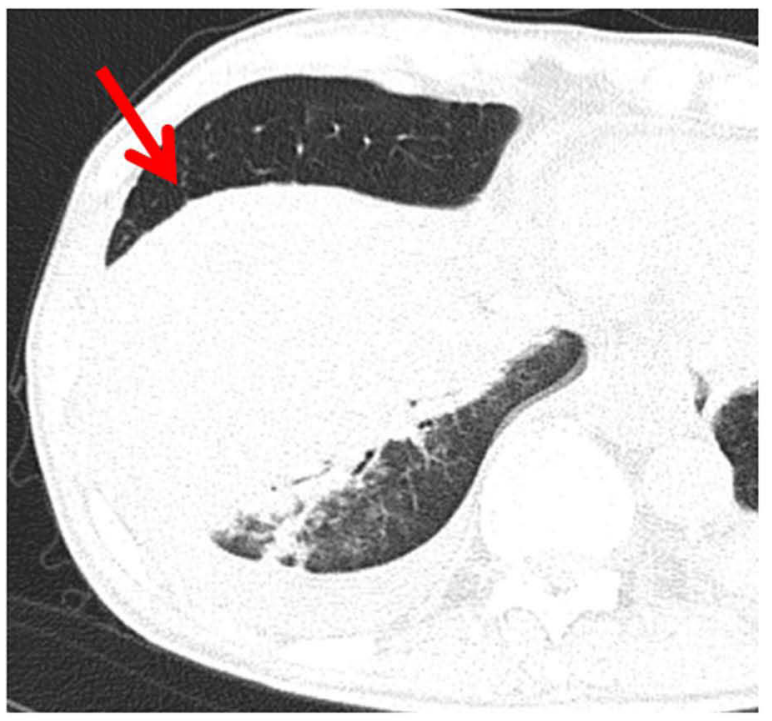

A

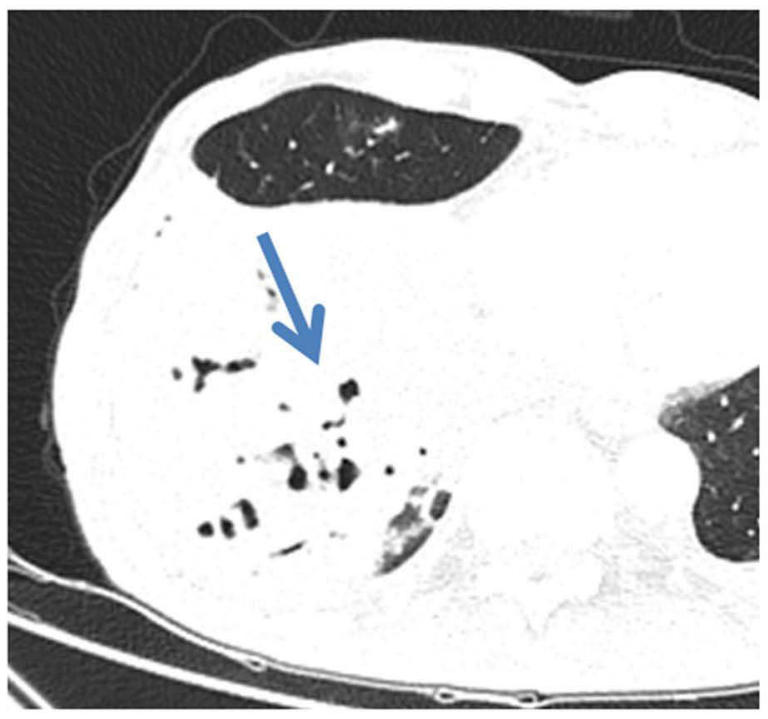

C

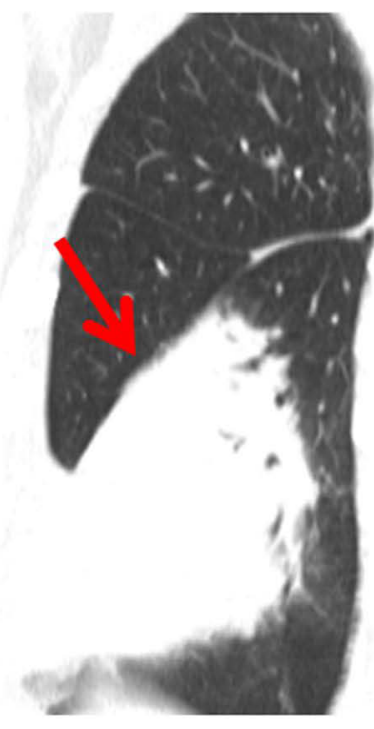

B

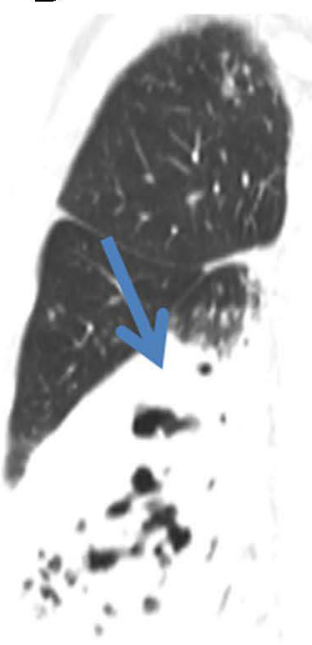

D

Figure 4 Pulmonary MALT lymphoma in a 68-year-old woman. (A and B) Thin-section CT and multi-planar reconstruction showed a consolidation with a bulging fissure (red arrow) in right lower lobe. (C and D) The patient received chemotherapy. However, I year later, chest CT imaging showed that area of the consolidation in lower right lobe became larger than before, and the dilated bronchus could be seen (blue arrow), which means the condition progressed.

Abbreviations: CT, computed tomography; MALT, mucosa-associated lymphoid tissue.

lobar pneumonia, and developed three logistic regression models to distinguish pulmonary MALT lymphoma from lobar pneumonia. The results demonstrated that age, mild clinical symptoms, bronchiectasis, and bulging of interlobar fissure demonstrated great potential in distinguishing lobar pneumonia from MALT lymphoma.

Patients with MALT lymphoma tend to present around the age of 60 , but there are also reports of young patients, while the patients with lobar pneumonia are mostly young and middle-aged, and the age of the patients are relatively young. In our study, the average age of MALT lymphoma patients was older than that of lobar pneumonia patients, consistent with previous studies. ${ }^{13}$ Previous studies have shown that nearly $30 \%$ of the patients with MALT lymphoma were asymptomatic, ${ }^{6}$ while most patients with lobar pneumonia have respiratory symptoms such as fever, cough, expectoration, dyspnea, chest pain, and chest distress. ${ }^{14}$ Our result also shown that the respiratory symptoms was more common in lobar pneumonia than MALT lymphoma of consolidation pattern $(p=0.002)$. Therefore, we speculate that this may be due to the most frequent pathogen of lobar pneumonia is Streptococcus pneumoniae, and the main 

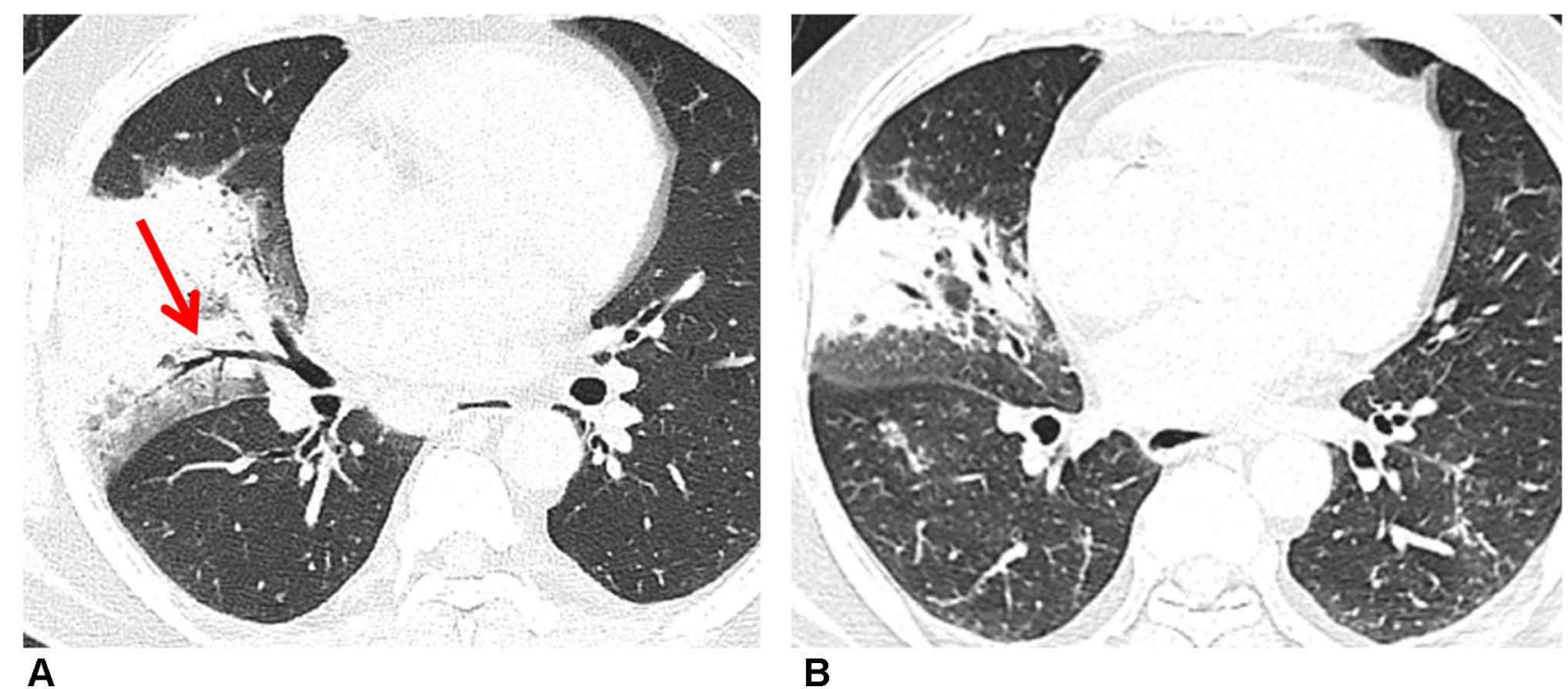

B

Figure 5 Lobar pneumonia in a 64-year-old man. (A) CT imaging showed consolidation with air bronchogram (red arrow) in the right middle lobe. (B) After two weeks of antibiotics treatment, thoracic CT showed the area of consolidation in the right middle lobe was reduced, which means the condition improved.

Abbreviation: $\mathrm{CT}$, computed tomography.

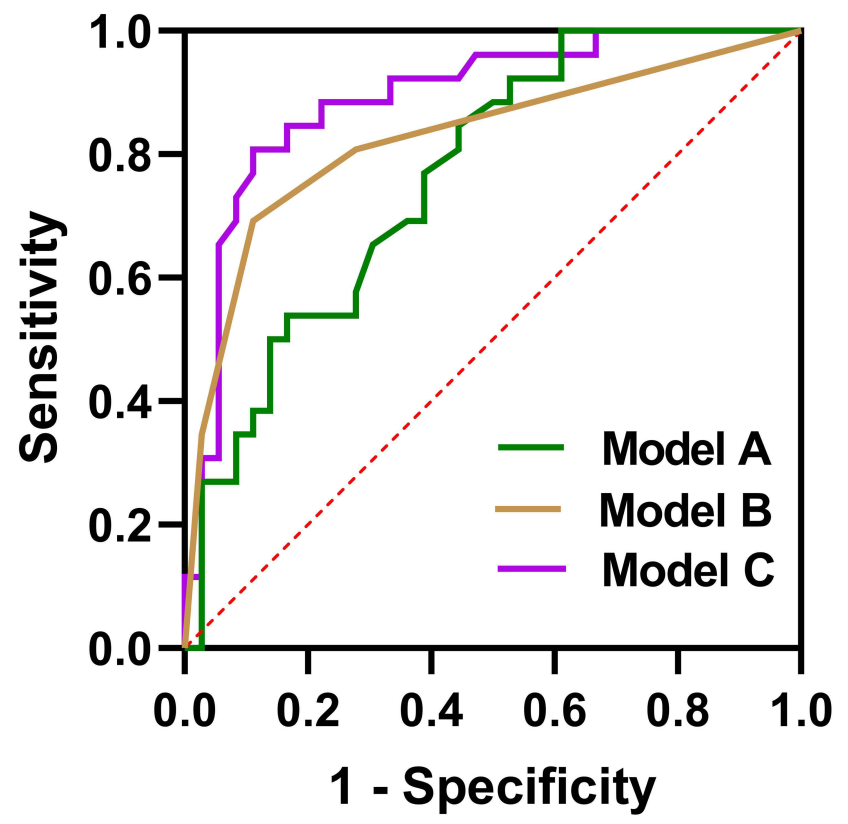

Figure 6 Receiver operating characteristic curves. Model A: age+respiratory symptoms, Model B: bronchiectasis + bulging of interlobar fissure, and Model C: age + respiratory symptoms + bronchiectasis + bulging of interlobar fissure.

pathogenic mechanism is allergic reaction. The young adults are highly reactive, once infected with Streptococcus pneumonia, the pathogenic bacteria proliferate rapidly and produce a large amount of exudate, so the clinical manifestations are more obvious. However, the elderly are poorly reactive, and the clinical manifestations are often atypical or covered by other symptoms.
Chest CT is the imaging modality of choice for evaluating pulmonary lesions, and in this study, all patients underwent chest CT scans. In both transverse and longitudinal distributions, most lobar pneumonia and MALT lymphoma cases were randomly distributed, with no predominant central distribution trend, which is consistent with previous studies. ${ }^{6}$

MALT lymphoma, pneumonia, and lung adenocarcinoma can all be manifested as chronic lung consolidations with air bronchogram sign. In MALT lymphoma, tumor cells infiltrated and grew along the bronchial submucosal epithelium and interstitial lung, while bronchial wall is inviolable, therefore, residual bronchus can be seen in the consolidation area. ${ }^{15}$ The consolidation in lobar pneumonia is caused by inflammatory cells and fluid filling the alveoli, and bronchial lumen can also stay smooth. In our study, there was no significant difference in air bronchogram sign between MALT lymphoma and lobar pneumonia $(88.5 \%$ vs $94.4 \%, p=0.39)$. We also found there was no significant difference in lesion size, calcification, mediastinum or hilar lymph nodes enlargement, and pleural effusion between MALT lymphoma and lobar pneumonia with $p>0.05$, consistent with previous studies.

We observed bronchiectasis within the consolidation was more frequent in patients with MALT lymphoma than those with lobar pneumonia $(69.2 \%$ vs $11.1 \%$, $p<0.001)$. Therefore, we speculate that bronchiectasis within the consolidation is one of relatively characteristic 
Table 3 Diagnostic Performance of Clinical Characteristics, Bronchiectasis, and Bulging of Interlobar Fissure in Differentiating Lobar Pneumonia from MALT

\begin{tabular}{|l|c|c|c|c|c|c|}
\hline Model & AUC (95\% Cl) & NPV (\%) & PPV (\%) & SEN (\%) & SPE (\%) & Accuracy (\%) \\
\hline Model A & $0.765(0.649-0.88 I)$ & 71.43 & 70.00 & 83.33 & 53.85 & 70.97 \\
Model B & $0.826(0.714-0.938)$ & 80.00 & 81.82 & 88.89 & 69.23 & 80.65 \\
Model C & $0.891(0.806-0.975)$ & 84.21 & 83.33 & 88.89 & 76.92 & 83.87 \\
\hline
\end{tabular}

Notes: Model A—age+respiratory symptoms; Model B—bronchomatic + bulging of interlobar fissure; Model C—age + respiratory symptoms + bronchomatic + bulging of interlobar fissure.

Abbreviations: MALT, mucosa-associated lymphoid tissue; AUC, area under the curve; $\mathrm{Cl}$, confidence interval; NPV, negative predictive value; PPV, positive predictive value; SEN, sensitivity; SPE, specificity.

CT manifestations of MALT lymphoma. ${ }^{16}$ It is different from the traditional bronchiectasis in that there is no bronchial wall destruction or tumor necrosis in the dilated bronchus. The pathological basis may result from the destruction of parenchyma adjacent to bronchus or alveolar collapse due to infiltration of lymphoid tissue, as well as the proliferation and stretching of surrounding fibrous tissue. $^{6}$

In our study, we also found bulging of interlobar fissure was more frequent in patients with MALT lymphoma than those with lobar pneumonia $(46.2 \%$ vs $19.4 \%, p=0.024)$. In pulmonary MALT lymphoma, the mucus secreted by tumor cells raises swelling of the lung lobes, leading to bulging of interlobular fissures. In the case of pneumonia, it is due to the secretion of inflammatory cells and body fluids that result in bulging of interlobular fissures. ${ }^{17}$

We constructed a logical model with clinical characteristics (age and respiratory symptoms) and CT manifestations (bronchomatic, and bulging of interlobar fissure) with $p$-value less than 0.05 . Our data indicated that the model could obtain high AUC of 0.891 , sensitivity of $88.89 \%$, specificity of $76.92 \%$, accuracy of $83.87 \%$, 83.33\% PPV, and 84.21\% NPV, suggesting that these features may help distinguish lobar pneumonia from pulmonary MALT lymphoma. Based on this result, we conclude that if these features are in favor of pneumonia, then patients can be treated with antibiotics, but if these features are in favor of MALT lymphoma, then a histologic confirmation should be done.

We know our study has several limitations. First, it was a retrospective study, which may imply selection bias. Second, due to some patients lack contrast-enhanced CT information, we only analyzed unenhanced CT scan in this study. Third, although the number of MALT lymphoma patients in our study was larger than most previous reports, the number of enrolled MALT lymphoma patients was still relatively small due to our strict inclusion criteria.
Therefore, further larger-cohort multicenter prospective program may be needed to confirm our results.

In conclusion, middle-aged, presence of mild clinical symptoms, bronchiectasis and bulging of the interlobar fissure on chest CT images present high diagnostic accuracy in distinguishing pulmonary MALT lymphoma from lobar pneumonia. These clinical characteristics and CT features can provide diagnostic clues and lead the clinician to make a reasonable treatment plan.

\section{Acknowledgments}

This work was supported by the National Natural Science Foundation of China (Grant No. 82001883, 81771899).

\section{Disclosure}

The authors report no conflicts of interest in this work.

\section{References}

1. Shinoda K, Taki H, Tsuda T, Hayashi R, Nomoto K, Tobe K. Primary pulmonary lymphoma presenting with multiple lung nodules. $\mathrm{Am}$ J Respir Crit Care Med. 2014;190(9):e30-e31. doi:10.1164/rccm.20 1401-0086IM

2. Bi W, Zhao S, Wu C, et al. Pulmonary mucosa-associated lymphoid tissue lymphoma: CT findings and pathological basis. J Surg Oncol. 2021;123(5):1336-1344. doi:10.1002/jso.26403

3. Sanguedolce F, Zanelli M, Zizzo M, et al. Primary pulmonary B-cell lymphoma: a review and update. Cancers. 2021;13(3):415. doi:10. 3390/cancers 13030415

4. Wang Y, Han J, Zhang F, et al. Comparison of radiologic characteristics and pathological presentations of primary pulmonary lymphoma in 22 patients. $J$ Int Med Res. 2020;48(4):300060519879854.

5. Chen Y, Chen A, Jiang H, et al. HRCT in primary pulmonary lymphoma: can CT imaging phenotypes differentiate histological subtypes between mucosa-associated lymphoid tissue (MALT) lymphoma and non-MALT lymphoma? J Thorac Dis. 2018;10(11):6040-6049. doi:10.21037/jtd.2018.10.63

6. Deng W, Wan Y, Yu JQ. Pulmonary MALT lymphoma has variable features on CT. Sci Rep. 2019;9(1):8657. doi:10.1038/s41598-01945144-9

7. Iftikhar A, Magh A, Cheema MA, Thappa S, Sahni S, Karbowitz S. Primary pulmonary MALT lymphoma presenting as non-resolving pneumonia. Adv Respir Med. 2017;85(4):202-205. doi:10.5603/ ARM.2017.0033 
8. Yao D, Zhang L, Wu PL, et al. Clinical and misdiagnosed analysis of primary pulmonary lymphoma: a retrospective study. BMC Cancer. 2018;18(1):281. doi:10.1186/s12885-018-4184-1

9. Kelemen K, Rimsza LM, Craig FE. Primary pulmonary B-cell lymphoma. Semin Diagn Pathol. 2020;37(6):259-267. doi:10.1053/ j.semdp.2020.04.002

10. Wang L, Ye G, Liu Z, et al. Clinical characteristics, diagnosis, treatment, and prognostic factors of pulmonary mucosa-associated lymphoid tissue-derived lymphoma. Cancer Med. 2019;8(18):76 60-7668. doi:10.1002/cam4.2683

11. Cozzi D, Dini C, Mungai F, Puccini B, Rigacci L, Miele V. Primary pulmonary lymphoma: imaging findings in 30 cases. Radiol Med. 2019;124(12):1262-1269. doi:10.1007/s11547-019-01091-z

12. Yan W, Wu B, Liao AJ, Yang W, Wang HH. Watch-and-wait or immediate immunotherapy/immunochemotherapy in patients with phase IE primary pulmonary MALT lymphoma? A multicenter retrospective study. Ann Hematol. 2021;100(3):709-714.

13. Zhao J, Wang H. Correlation between 18F-FDG PET/CT semiquantitative parameters and $\mathrm{Ki}-67$ expression in pulmonary mucosa-associated lymphoid tissue lymphoma. J Med Imaging Radiat Oncol. 2021;65(2):188-194. doi:10.1111/1754-9485.13146
14. Chang CJ, Chiu NC, Huang FY, et al. Predictive value of Thomsen-Friedenreich antigen activation for Streptococcus pneumoniae infection and severity in pediatric lobar pneumonia. J Microbiol Immunol Infect. 2019;52(4):571-577. doi:10.1016/j. jmii.2017.08.011

15. Albano D, Durmo R, Treglia G, Giubbini R, Bertagna F. 18F-FDG $\mathrm{PET} / \mathrm{CT}$ or PET role in MALT lymphoma: an open issue not yet solved-a critical review. Clin Lymphoma Myeloma Leuk. 2020;20 (3):137-146. doi:10.1016/j.clml.2019.10.006

16. Kinsely BL, Mastey LA, Mergo PJ, et al. Pulmonary mucosa-associated lymphoid tissue lymphoma: CT and pathologic findings. AJR Am J Roentgenol. 1999;172(5):1321-1326. doi:10.22 14/ajr.172.5.10227510

17. Nambu A, Ozawa K, Kobayashi N, Tago M. Imaging of community-acquired pneumonia: roles of imaging examinations, imaging diagnosis of specific pathogens and discrimination from noninfectious diseases. World J Radiol. 2014;6(10):779-793. doi:10. 4329/wjr.v6.i10.779

\section{Publish your work in this journal}

Cancer Management and Research is an international, peer-reviewed open access journal focusing on cancer research and the optimal use of preventative and integrated treatment interventions to achieve improved outcomes, enhanced survival and quality of life for the cancer patient.
The manuscript management system is completely online and includes a very quick and fair peer-review system, which is all easy to use. Visit http://www.dovepress.com/testimonials.php to read real quotes from published authors. 